\title{
The CRASH Project: Defect Detection and Classification in Ferrite Cores
}

Massimo Mari ${ }^{1}$, Carlo Dambra ${ }^{1}$, Dmitry Chetverikov ${ }^{2}$, Judit Verestoy ${ }^{2}$, Adam Jozwik ${ }^{3}$, Mariusz Nieniewski ${ }^{4}$, Leszek Chmielewski ${ }^{5}$, Marek Sklodowski ${ }^{5}$, Waldemar Cudny ${ }^{5}$, Martin Lugg ${ }^{6}$

1 University of Trento, Laboratorio di Ingegneria Informatica, Via F. Zeni 8, I-38068, Rovereto ITALY,

Phone +3910 3532662, Fax +3910 3532134, e-mail: mate@dibe.unige.it

2 Hungarian Academy of Science, Computer and Automation Research Institute, Kende u.13-17, 1111, Budapest HUNGARY,

Phone +361181 1760, Fax +3611667503, e-mail mitya@leader.ipan.sztaki.hu

3

Polish Academy of Sciences, Institute of Biocybernetics and Biomedical Engineering, Trojdena 4, Warsaw POLAND,

Phone +48 226599 143, Fax +48 22 6597030, e-mail adamj@ibbrain.ibb.waw.pl

4 Polish Academy of Sciences, Department of Fundamental Research in Electrical Eng., Swietokrzyska 21, 00049 Warsaw POLAND,

Phone +48 22 8261281, Fax +4822 8269802, e-mail: mnieniew@ippt.gov.pl

5 Association for Image Processing, IFTR PAS,

Swietokrzyska 21, 00049 Warsaw POLAND,

Phone +48 22 8261281, Fax +48 22 8269802, e-mail: lchmiel@ippt.gov.pl

6 Technical Software Consultant Ltd., 6 Mill Square, Featherstone Road,

Wolwerton Mill, MK 125RB Milton keynes, UK

Phone +44 1908220 255, Fax +44 1908 220959, e-mail martinl@tscuk.demon.co.uk

\begin{abstract}
The paper presents a work developed in the framework of the two years COPERNICUS technological research project CRASH (CRack and SHape defect detection in ferrite cores) CIPA-CT94 0153, in progress since 1995.

The CRASH project concerns automated quality inspection of ferrite cores. CRASH studies the development of optical and electromagnetic systems that may be integrated in a working module to increase the recognition of imperfections on ferrite materials. Analysis and processing of acquired images and signals, as well as specification of ad-hoc algorithms for classification purposes constitute the technical approach to the problem. The achieved results show the capability of the system to detect different kind of imperfections in ferrite cores (shape defects, surface defects and subsurface imperfections) and classify them with low error rates.

After an introduction to the problem in Section 1, different techniques of defect detection with different sensors are shown in Section 2, and Section 3 describes the achieved classification results.
\end{abstract}




\section{Introduction}

The general objectives of the CRASH project [1] is to select an develop a system for automated quality inspection of industrially produced objects, with special attention paid to ferrite cores, to solve the problem of manual quality control that affects many factories especially in the Eastern Countries.

The main requirements of the novel system are the classification of different ferritic cores and the detection of dimensional and surface defects which greatly affect the quality of materials. To detect ferrite properties, dimensional defects (shape defects i.e.) and surface defects (surface cracks), two different sensors have been used: an optical sensor and an electromagnetic sensor in a new precision system.

In the inspection of surface defects, for faster processing, a two-level approach has been used, on the basis of the acquired images with the specially designed sensor. The first processing level is the detection of regions where irregularities could occur; the second level is the classification of pixels which belong to potential defects. Feature-based pattern recognition method of $k$ - Nearest Neighbours (k-NN) is used to classify a pixel as belonging to one of the classes of defects or to a good surface.

\section{Defect Detection}

The proposed system uses two different inspection sensors on the basis that complementary information from inspection based on different physical principles enhance confidence in the inspection results. Different techniques have been used for different kind of imperfections, following the users requirements: shape defects (optical) and surface defects (both optical and electromagnetic).

\subsection{Shape Defects Detection and Measurements}

The current practice of core production control is to select sample cores and measure their dimensions with a mechanical gauge which either accepts or rejects the core. To comply with this practice, an optical shape gauge system has been developed in order to quickly reject the obviously defective cores. The system measures the less obvious but still critical dimensional deviations from the standard values specified by the producer and represented by a reference digital image and a tolerance image, compared to the shape being measured. An important feature of the shape gauge system is its capability to handle arbitrarily oriented projections of the 3D objects. Therefore, the comparison of the measured and the reference shape is shift and rotation invariant. In figure 1 an example of the reference image, the measured and difference images are shown for a defective E-core (bidimensional view). 


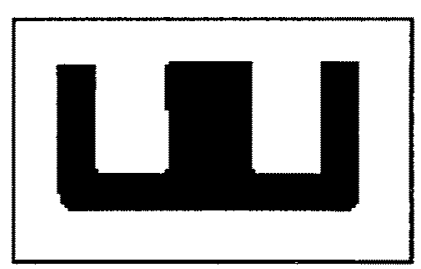

a
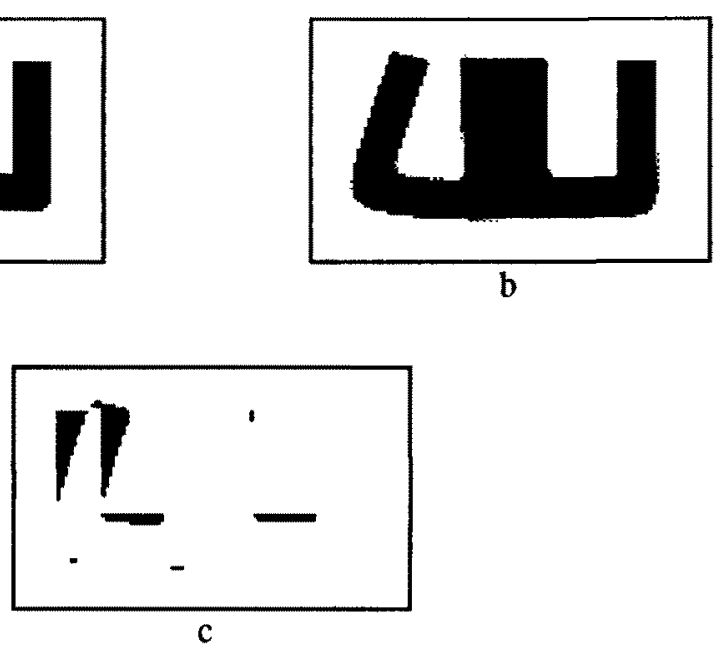

Fig. 1. A bidimensional view of a) reference image, b) measured image, c) difference image.

A novel shape representation, the polar run-length code (PRLC), was introduced that is particularly suitable for this purpose.

Under these conditions, the optical gauge system: (a) rejects the core if the overall deviation is above a threshold; (b) measures the dimensions of the core; (c) detects and measures selected types of shape defect; (d) keeps track of the major statistics for production control.

Experimental measures have been done on a large number of sample cores, both defective and presumably good, to demonstrate the capability of the system.

\subsection{Surface Core Inspection with Electromagnetic Sensor}

To complement the optical inspection of ferrite cores for shape distortion, an electromagnetic technique is needed to find other kind of imperfections, such as narrow surface breaking cracks and sub-surface voids.

The AC based techniques [2] are not suitable because the very high resistivity of the ferrite prevented any appreciable induction of current. Various other non-contacting techniques, such as conventional Eddy currents, $\mathrm{AC}$ and $\mathrm{DC}$ flux leakage, suffer from the same problem. For the detection of surface cracks in ferrite cores, the contacting DC Potential Drop technique (DCPD) has been used. This proved very easy to implement, because the high resistivity gives rise to high, easily measurable, surface potential differences. Surface maps of potential differences, both parallel and perpendicular to the input electric field, were collected by an array of contacting sprung pins via a multiplexer, which was scanned along the core surface. The peaks and troughs detected in these maps indicated the positions of defects, and the signal amplitudes could be related to defect depth using a simple model of potential distribution in the component [2]. From the information of each digital map, a suitable set of features is then extracted and given as input to the classifier (see Section 3). 


\subsection{Surface Defect Detection with Optical Sensor}

The grey level images of the illuminated cores are acquired through the use of a specially designed LED illuminating system. These images are analysed by a defect detection system [3] which consists of: a morphological defect detector, a morphological pyramid, and a data reduction software. Fig. 2 shows an example of the pyramid obtained for a defective core. The images on the left side show successive levels of the original image, whereas the images on the right show grey level maps of defects. As can be observed, by reducing the resolution one is able to detect bigger and bigger defects. The maps of defects are then used by the defect classification software.

The obtained results covered a large range of defects and were quite satisfactory. A set of most informative features is then extracted from each pattern of single pixel; in particular, as the features, the following functions defined on a suitable neighbourhood of each pixel have been used: raw brightness, standard deviation, absolute value of gradient and texture measures according to [4], [5] and [6].
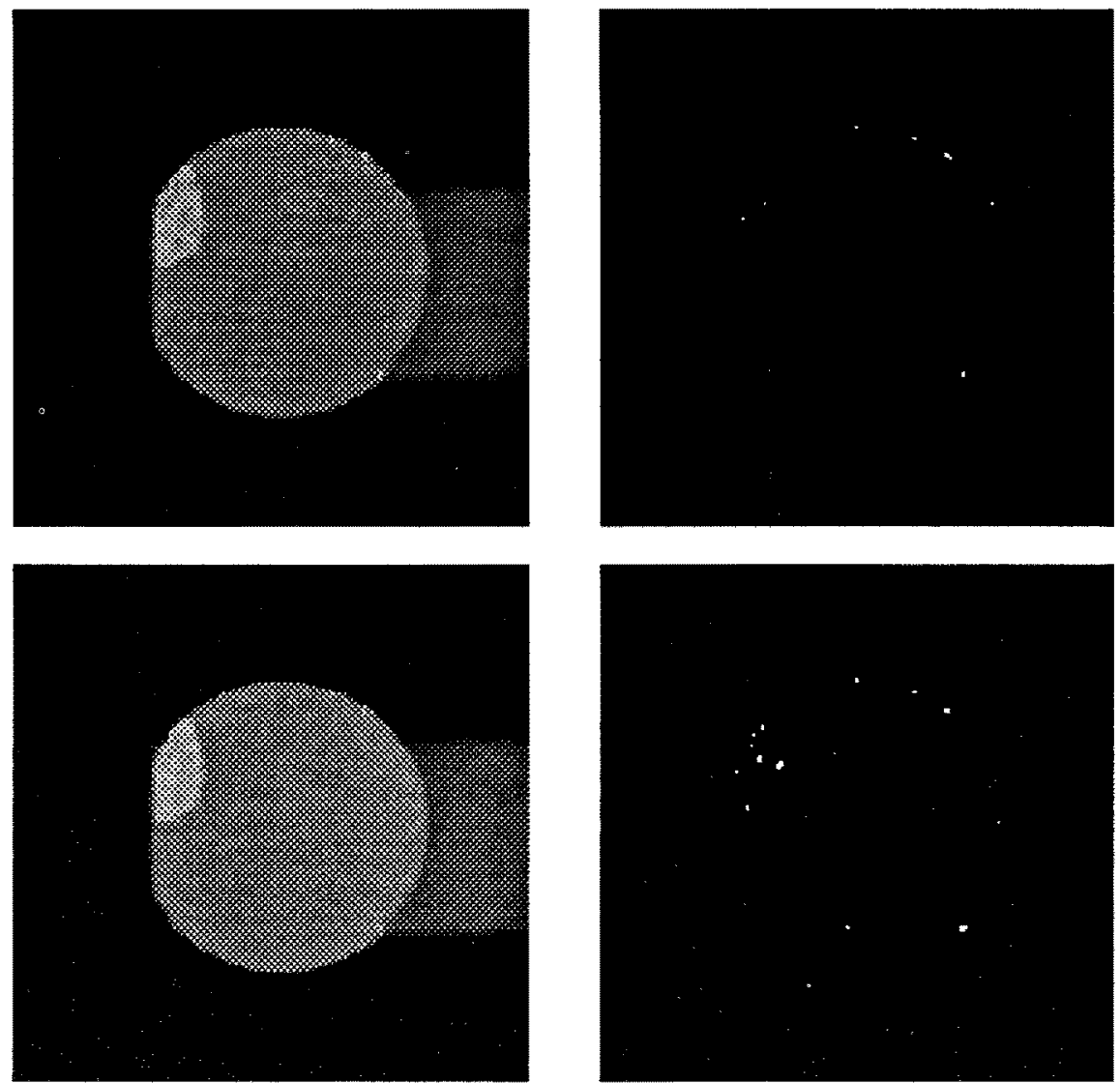

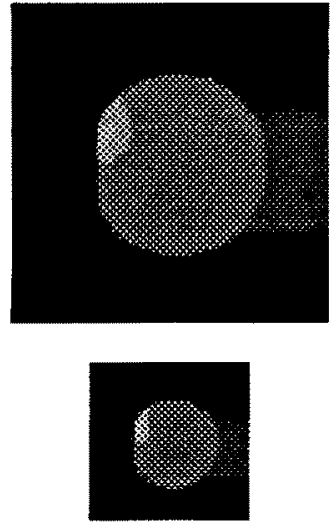
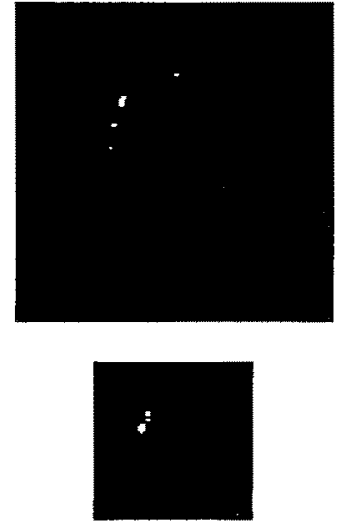

Fig. 2. Morphological pyramid for a defective ferrite core (the relative size is not observed. Each successive level of the pyramid is two times smaller than the previous one).

\section{Classification Results}

In visual inspection of cores surfaces one of the approaches is to recognise whether a given pixel of a core image belong to a "good" surface, to the background or to the one of considered types of irregularities. Basing on the result of such classification carried out for all pixels, a global rule for core classification can be obtained. For the classification of surface defects (optical sensor) the following five classes of pixels have been considered: 1.good object, 2.background, 3.chipping, 4.chipping with external edge, 5. chipping with internal edge. The classification rule can be created on the basis of the set of pixels with a known class membership. In our case the training set contains 71406 pixels. In Fig. 3 an image of a core with marked training pixels and the classes of pixels for training the classifier is shown.

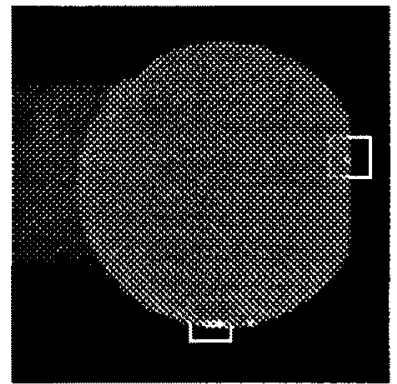

a

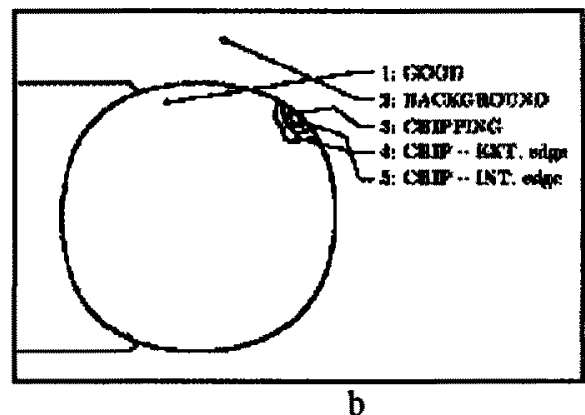

b

Fig. 3. a) a core with marked training pixels; b) classes of pixels for training the $k-N N$ classifier. 
To construct a decision rule for classification of pixels, a method based on the k-NN (k - Nearest Neighbours) technique [7] has been used. With respect to other approaches, for instance artificial neuron like methodology that are in fashion now, the learning of $\mathbf{k}-\mathrm{NN}$ classifiers can be very fast realised. As an example of classification results, in Table 1 the misclassification matrix shows that a maximum error rate of 0.27 for the optical defect detection method has been obtained on a significative test set of cores.

\begin{tabular}{|c|c|c|c|c|c|}
\hline Classes & 1 & 2 & 3 & 4 & 5 \\
\hline 1 & 0.8960 & 0.0082 & 0.0186 & 0.0635 & 0.0137 \\
\hline 2 & 0.0018 & 0.9753 & 0.0000 & 0.0001 & 0.0228 \\
\hline 3 & 0.0476 & 0.0009 & 0.6465 & 0.2701 & 0.0349 \\
\hline 4 & 0.0604 & 0.0000 & 0.0877 & 0.8250 & 0.0269 \\
\hline 5 & 0.0048 & 0.0116 & 0.0048 & 0.0029 & 0.9759 \\
\hline
\end{tabular}

Table 1. The misclassification matrix: probabilities that object from the class " $\mathrm{i}$ " (row) is assigned to the class " $\mathrm{j}$ " (column) for five classes of optical method.

As regards the surface defect (crack) detection through the electromagnetic sensor, the application of standard error measures of False Positive (FP) and False Negative (FN) [8] to the classified pixels has given a maximum error percentage value of $0.045 \%$ (FP) and $0.038 \%$ (FN) for a test set of surface cracked samples.

A feature selection strategy is strongly recommended since it may not only simplify the classification but also significantly improve it.

As conclusion, the project results will be applicable to a class of quality inspection problems for various objects, not only these selected as prototypes in this paper. The inspection and measuring system that the CRASH research has developed would constitute an essential element of a future Computer Integrated Manufacturing (CIM) module to the overall production of ferritic cores. This would greatly enhanced the productivity and quality control of the industrial partners. A significative contribution has been given form the CRASH activity to the standards of the IEC (International Electrotechnical Commission) for quality assessment of ferrite cores (Technical Committee TC51 of IEC). 


\section{References}

1. The CRASH project official home page is at http://www.lii.unitn.it The CRASH Consortium:

UNT University of Trento, Rovereto ITALY - Project Coordinator

HAS Hungarian Academy of Sciences, Budapest HUNGARY

PAS Polish Academy of Sciences, Warsaw POLAND

AIP Association of Image Processing, Warsaw POLAND

TSC Technical Software Consultant Ltd., Milton Keynes UK

POLFER POLFER Magnetic Materials, Warsaw POLAND.

2. D. Mirshekar-Syahkal, R. Collins and D. H. Michael, "Developments in surface cracks by the A.C. field technique", Review of progress in quantitative NDT Evaluation, Donald O. Thompson et al. eds., Plenum Publishing, 1985, pp. 349-357.

3. M. Nieniewski, Morphological Method of Detection of defects on the Surface of Ferrite Cores, Proc. 10th Scandinavian Conference on Image Analysis, Lappeenranta, Jun 1997.

4. K. I. Laws, Textured image segmentation, Univ. of Southern California, Image Processing Institute, USCIPI Report 940, Jan 1980.

5. W. K. Pratt, Digital Image Processing, John Wiley, New York 1991.

6. C-M. Wu, Y-C. Chen, Statistical feature matrix for texture analysis, CVGIP: Graphical Models and Image Processing, 54, 5, 1992, pp. 407-419.

7. A.Jozwik, L.Chmielewski, W.Cudny, M.Sklodowski, A 1-NN preclassifier for fuzzy k-NN rule, Proc. 13th Int. Conf. Pattern Recognition, ICPR96, Wien, Austria, August 1996, Track D, pp. $234-238$.

8. Z. Wu, "Homogeneity testing for unlabeled data: a performance evaluation", Graphical Models and Image Processing, vol.55, September 1993, pp.370380. 